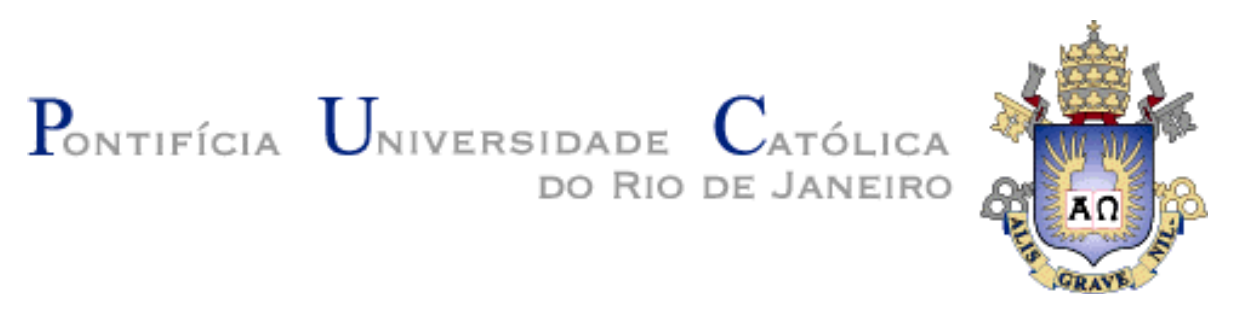

Tulio Jorge de Alcântara Neves de Souza Anibolete

\title{
Boosting para \\ Sistemas de Recomendação
}

Dissertação de Mestrado

Dissertação apresentada como requisito parcial para obtenção do título de Mestre pelo Programa de PósGraduação em Informática da PUC-Rio.

Orientador: Prof. Ruy Luiz Milidiú

Rio de Janeiro

Julho de 2008 


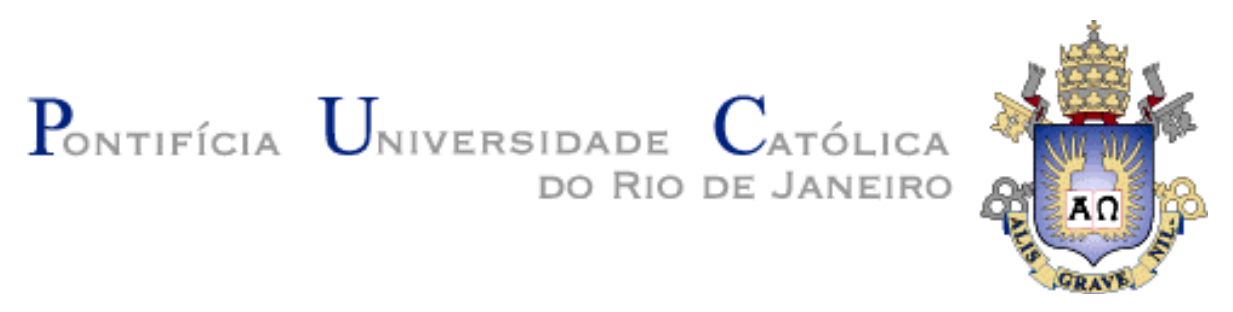

Tulio Jorge de Alcântara Neves de Souza Anibolete

\title{
Boosting para \\ Sistemas de Recomendação
}

Dissertação apresentada como requisito parcial para obtenção do título de Mestre pelo Programa de PósGraduação em Informática da PUC-Rio. Aprovada pela Comissão Examinadora abaixo assinada.

\author{
Prof. Ruy Luiz Milidiú \\ Orientador \\ Departamento de Informática - PUC-Rio \\ Prof. Daniel Schwabe \\ Departamento de Informática - PUC-Rio
}

Prof. Marcus Vinicius Soledade Poggi de Aragão

Departamento de Informática - PUC-Rio

Prof. José Eugenio Leal

Coordenador(a) Setorial do Centro Técnico Científico - PUC-Rio

Rio de Janeiro, 30 de julho de 2008 
Todos os direitos reservados. É proibida a reprodução total ou parcial do trabalho sem autorização da universidade, do autor e do orientador.

\section{Tulio Jorge de Alcântara Neves de Souza Anibolete}

Recebeu seu título de Bacharel em Informática pela Pontifícia Universidade Católica do Rio de Janeiro (PUC-Rio) em 2004. Desde 2007, integra, na mesma instituição, a equipe de pesquisade Sistemas de Recomendação, do Laboratório de Engenharia de Algoritmos e Redes Neurais (LEARN). Possui 4 anos de experiência profissional atuando como analista de sistemas e trabalha no Banco Nacional de Desenvolvimento Econômico e Social (BNDES).

Ficha Catalográfica

Anibolete, Tulio Jorge de Alcântara Neves de Souza

Boosting para Sistemas de Recomendação / Tulio Jorge de Alcântara Neves de Souza Anibolete; orientador: Ruy Luiz Milidiú. - Rio de Janeiro: PUC, Departamento de Informática, 2008.

60 f. : Il. (col.) ; 29,7 cm

1. Dissertação (mestrado) - Pontifícia Universidade Católica do Rio de Janeiro, Departamento de Informática.

Inclui referências bibliográficas.

1. Informática - Teses. 2. Filtragem Colaborativa. 3. Aprendizado de Máquina. 4. Sistemas de Recomendação. 5. Boosting. I. Milidiú, Ruy Luiz. II. Pontifícia Universidade Católica do Rio de Janeiro. Departamento de Informática. III. Título. 


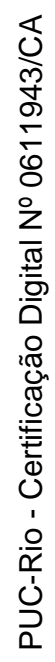

Às minhas queridas tias Márcia e lara. 


\section{Agradecimentos}

O maior agradecimento deste trabalho vai para as minhas tias pelo carinho, dedicação e apoio.

Agradeço também ao meu orientador, Prof. Ruy Luiz Milidiú, pelo incentivo, liderança e sugestão do tema deste trabalho.

O agradecimento seguinte vai para os professores Ivan Mathias Filho e Sérgio Colcher, pela confiança em mim depositada ao me recomendarem para o mestrado e para os professores Daniel Schwabe e Marcus Vinícius Soledade Poggi de Aragão, por aceitarem participar da banca examinadora.

Não posso me esquecer dos meus amigos do LEARN e todos os outros conquistados na PUC-Rio, aos quais agradeço por consolidarem um ambiente repleto de idéias criativas: Roberto Cavalcante, Diogo Mendonça, Leonardo Majowka, Cícero Nogueira, Júlio Duarte, Sérgio Ciglione, Andrew Diniz, Bruno Siqueira, Rodnei Couto, Márcio Aguiar e Eduardo Fonseca.

Agradeço, ainda, aos meus grandes amigos, Allan Miranda, Shirley Arruda e José Carlos Seraphim Jr. pelos conselhos nas horas adequadas, energia e estímulo nas horas em que mais precisei. 


\section{Resumo}

Anibolete, Tulio Jorge de Alcântara Neves Souza; Milidiú, Ruy Luiz. Boosting para Sistemas de Recomendação. Rio de Janeiro, 2008. 60p. Dissertação de Mestrado - Departamento de Informática, Pontifícia Universidade Católica do Rio de Janeiro.

Com a quantidade de informação e sua disponibilidade facilitada pelo uso da Internet, diversas opções são oferecidas às pessoas e estas, normalmente, possuem pouca ou quase nenhuma experiência para decidir dentre as alternativas existentes. Neste âmbito, os Sistemas de Recomendação surgem para organizar e recomendar automaticamente, através de Aprendizado de Máquina, itens interessantes aos usuários. Um dos grandes desafios deste tipo de sistema é realizar o casamento correto entre o que está sendo recomendado e aqueles que estão recebendo a recomendação. Este trabalho aborda um Sistema de Recomendação baseado em Filtragem Colaborativa, técnica cuja essência está na troca de experiências entre usuários com interesses comuns. Na Filtragem Colaborativa, os usuários pontuam cada item experimentado de forma a indicar sua relevância, permitindo que outros do mesmo grupo se beneficiem destas pontuações. Nosso objetivo é utilizar um algoritmo de Boosting para otimizar a performance dois Sistemas de Recomendação. Para isto, utilizamos uma base de dados de anúncios com fins de validação e uma base de dados de filmes com fins de teste. Após adaptações nas estratégias convencionais de Boosting, alcançamos melhorias de até $3 \%$ sobre a performance do algoritmo original.

\section{Palavras-chave}

Boosting, Filtragem Colaborativa, Sistemas de Recomendação, Aprendizado de Máquina 


\section{Abstract}

Anibolete, Tulio Jorge de Alcântara Neves de Souza; Milidiú, Ruy Luiz (Advisor). Boosting for Recommendation Systems. Rio de Janeiro, 2008. 60p. MSc. Dissertation - Departamento de Informática, Pontifícia Universidade Católica do Rio de Janeiro.

With the amount of information and its easy availability on the Internet, many options are offered to the people and they, normally, have little or almost no experience to decide between the existing alternatives. In this scene, the Recommendation Systems appear to organize and recommend automatically, through Machine Learning, the interesting items. One of the great recommendation challenges is to match correctly what is being recommended and who are receiving the recommendation. This work presents a Recommendation System based on Collaborative Filtering, technique whose essence is the exchange of experiences between users with common interests. In Collaborative Filtering, users rate each experimented item indicating its relevance allowing the use of ratings by other users of the same group. Our objective is to implement a Boosting algorithm in order to optimize a Recommendation System performance. For this, we use a database of advertisements with validation purposes and a database of movies with testing purposes. After adaptations in the conventional Boosting strategies, improvements of $3 \%$ were reached over the original algorithm.

\section{Keywords}

Boosting, Collaborative Filtering, Recommendation Systems, Machine Learning 


\section{Sumário}

1 Introdução 12

1.1. Motivação 12

1.1.1. Desafio da Recomendação de Anúncios na Internet 14

1.1.2. Desafio da Recomendação de Filmes 16

1.2. Trabalhos Relacionados 18

1.3. Objetivo 20

1.4. Organização do Trabalho 21

2 Boosting 22

2.1. Problema de Classificação 22

2.2. Validação Cruzada 23

2.3. AdaBoost 25

3 Sistemas de Recomendação e Filtragem Colaborativa 30

3.1. Recomendação Colaborativa 30

3.2. Fatoração de Matrizes 33

3.3. Adaptação do AdaBoost 34

4 Experimentos $\quad 37$

4.1. Conjunto de Dados de Anúncios 37

4.1.1. Geração da Base Artificial 38

4.2. Conjunto de Dados de Filmes 39

4.2.1. Segmentação dos Dados 39

4.3. Metodologia e Métricas de Avaliação 41

4.4. Resultados do algoritmo MM para Anúncios 42

4.5. Resultados do algoritmo FM para Anúncios 43

4.6. Resultados do Boosting com algoritmo FM para Anúncios 45

4.7. Resultados do algoritmo MM para Filmes 49

4.8. Resultados do algoritmo FM para Filmes $\quad 50$

4.9. Resultados do Boosting com algoritmo FM para Filmes 51 
5 Considerações Finais $\quad 55$

5.1. Contribuições 55

5.2. Trabalhos Futuros $\quad 56$

6 Referências Bibliográficas $\quad 57$ 


\section{Lista de figuras}

Figura 1: Motivação para os Sistemas de Recomendação. 12

Figura 2: Recomendação colaborativa a partir da experiência de outrem. 13

Figura 3: Lucro obtido através do clique nos anúncios. 15

$\begin{array}{ll}\text { Figura 4: Logística de aluguel da Netflix. } & 16\end{array}$

Figura 5: Ponto de partida do trabalho. 20

Figura 6: Objetivo do trabalho. 20

Figura 7: Exemplo de validação cruzada. $\quad 24$

Figura 8: Exemplo de repeated holdout. 24

Figura 9: Algoritmo AdaBoost. 27

Figura 10: Distribuição dos exemplos na classe “+” ou na classe “-“. 28

Figura 11: Executando a primeira rodada do AdaBoost. 28

Figura 12: Executando a segunda rodada do AdaBoost. 29

Figura 13: Executando a terceira e última rodada do AdaBoost. 29

Figura 14: Combinando os resultados. 29

Figura 15: Grupo de filmes correlatos. 31

Figura 16: Exemplificação da recomendação de filmes. 32

Figura 17: Algoritmo AdaBoost original para problemas regressivos. 35

Figura 18: Algoritmo AdaBoost adaptado para a recomendação. 36

Figura 19: Métricas de posição em função das épocas de treinamento. 44

Figura 20: Calibrando o AdaBoost.RS para anúncios. 46

Figura 21: Melhora de $\underline{\mathbf{3 \%}}$ no RMSE em relação ao FM. 48

Figura 22: Melhora de 2,5\% no EAMP e 0,8\% na PP em relação ao FM. 48

Figura 23: Calibrando o AdaBoost.RS para filmes. 52

Figura 24: Melhora de 0,2\% e 0,13\% no RMSE com relação ao FM. 54

Figura 25: Ranking da competição Netflix. 


\section{Lista de tabelas}

Tabela 1: Conversão da Posição em CTR Relativo. 38

Tabela 2: Clusters Utilizados na Recomendação de Filmes. 40

Tabela 3: Resultados do Sistema de Referência. 42

Tabela 4: Resultados do FM. 43

Tabela 5: Calibração do Fator de Suavização para Anúncios. 45

Tabela 6: Resultados do Boosting aplicado ao FM. 47

Tabela 7: Resultados do MM sobre os Clusters. 49

Tabela 8: Resultados do FM sobre o Cluster 1. 50

Tabela 9: Resultados do FM sobre o Cluster 2. 50

Tabela 10: Calibração do Fator de Suavização para Filmes. 51

Tabela 11: Resultados do Boosting aplicado ao FM no Cluster 1. 53

Tabela 12: Resultados do Boosting aplicado ao FM no Cluster 2. 